\title{
Clinical efficacy, safety and tolerability of Aliskiren Monotherapy (AM): an umbrella review of systematic reviews
}

\author{
Qiyuan Zhao ${ }^{1}$, Jiantong Shen ${ }^{1,2^{*}}$, Jingya Lu', Qi Jiang ${ }^{1}$ and Yuanyuan Wang ${ }^{1}$
}

\begin{abstract}
Background: Aliskiren is a newly developed drug. Its role in lowering BP has been recognized. However, the role of aliskiren in treating heart and renal diseases are still controversial.

Objective: To evaluate the existing evidence about clinical efficacy, safety and tolerability of aliskiren monotherapy (AM).

Methods: An umbrella review of systematic reviews of interventional studies. We searched Pubmed, Embase and Cochrane Library up to June 2019. Two reviewers applied inclusion criteria to the select potential articles independently. The extract and analyze of accessible data were did by two reviewers independently too. Discrepancies were resolved with discussion or the arbitration of the third author.

Results: Eventually, our review identified 14 eligible studies. Results showed that for essential hypertension patients, aliskiren showed a great superiority over placebo in BP reduction, BP response rate and BP control rate. Aliskiren and placebo, ARBs or ACEls showed no difference in the number or extent of adverse events. For heart failure patients, AM did not reduce BNP levels (SMD $-0.08,-0.31$ to 0.15 ) or mortality rate (RR 0.76, 0.32 to 1.80 ), but it decreased NT-proBNP (SMD -0.12, -0.21 to -0.03 ) and PRA levels (SMD 0.52, 0.30 to 0.75), increased PRC levels (SMD -0.66, -0.8 to -0.44 ). For patients who are suffered from hypertension and diabetes and/or nephropathy or albuminuria at the same time, aliskiren produced no significant effects (RR 0.97, 0.81 to 1.16 ).
\end{abstract}

Conclusion: We found solid evidence to support the benefits of aliskiren in the treatment of essential hypertension, aliskiren can produce significant effects in lowering BP and reliable safety. However, the effects of aliskiren in cardiovascular and renal outcomes were insignificant.

Trial registration: Study has been registered in PROSPERO (CRD42019142141).

Keywords: Aliskiren, Monotherapy, Clinical effectiveness, Safety

\section{Background}

Hypertension, as a highly prevalent disease and its control is still unsatisfactory. The prevalence of hypertension in the United States (defined as taking antihypertensive medication or having a systolic pressure of $\geq 140 \mathrm{mmHg}$ and/or a

\footnotetext{
*Correspondence: sjiantong@163.com

'School of Nursing, Huzhou University, Huzhou Central Hospital, 759 Erhuan Rd, Huzhou, 313000 Zhejiang, People's Republic of China

${ }^{2}$ School of Medicine, Huzhou University, Huzhou Central Hospital, 759 Erhuan Rd, Huzhou, 313000 Zhejiang, People's Republic of China
}

diastolic pressure $\geq 90 \mathrm{mmHg}$ ) was approximately $32 \%$ and had remained relatively constant since 1999 [1-3]. HF (heart failure) is also a rapidly growing public health problem, the estimated prevalence of it is $>37.7$ million individuals globally, it has creating a great burden to society [4].

By targeting blood pressure (BP) and related abnormalities of the heart and blood vessels, renin-angiotensinaldosterone system (RAAS) inhibitors can prevent target organ damage and related cardiovascular events [5]. Blockade of renin has already been recognized as the

(c) The Author(s). 2020 Open Access This article is licensed under a Creative Commons Attribution 4.0 International License which permits use, sharing, adaptation, distribution and reproduction in any medium or format, as long as you give appropriate credit to the original author(s) and the source, provide a link to the Creative Commons licence, and indicate if changes were made. The images or other third party material in this article are included in the article's Creative Commons licence, unless indicated otherwise in a credit line to the material. If material is not included in the article's Creative Commons licence and your intended use is not permitted by statutory regulation or exceeds the permitted use, you will need to obtain permission directly from the copyright holder. To view a copy of this licence, visit http://creativecommons.org/licenses/by/4.0/ The Creative Commons Public Domain Dedication waiver (http://creativecommons.org/publicdomain/zero/1.0/) applies to the data made available in this article, unless otherwise stated in a credit line to the data. 
optimal means for inhibiting the RAAS [6]. Aliskiren is the first one in a new class of oral, non-peptide, low molecular weight direct renin inhibitors (DRI) [7]. It has already been approved by the US Food and Drug Administration (FDA) and some European countries for the treatment of essential hypertension [8]. As reported, aliskiren produced good results on certain surrogate end-points in HF setting: BNP (brain natriuretic peptide) levels, Nterminal prohormone of BNP (NT-proBNP) levels, plasma renin activity (PRA), plasma renin concentration (PRC), etc [9] Aliskiren is also reported to have good effects on the renal function, as it can increase renal blood flow, and may prevent the deterioration of renal $[10,11]$.

Systematic reviews, meta-analyses and pooled analyses of interventional studies have evaluated the efficacy, safety and tolerability of aliskiren. However, in contrast with the promising prospect, several reviews showed that aliskiren presented no significant influence on several important clinical outcomes. In the present work, we carried out an umbrella review of the evidence across existing systematic reviews, meta-analyses and pooled analyses of interventional studies that reported clinical outcomes after using aliskiren monotherapy (AM).

Our overview was aimed to provide an overview of the range and validity of the reported associations between AM and clinical efficacy as well as the side effects. We compared aliskiren with placebo and other pharmaceutical drugs: Angiotensin Receptor Blockers (ARBs), AngiotensinConverting Enzyme Inhibitors (ACEIs), hydrochlorothiazide (HCT/HCTZ), etc. to evaluate the effects of cardiovascular outcomes and renal effects between AM and other antihypertensive (BP reduction, $\mathrm{BP}$ response rate, $\mathrm{BP}$ control rate, the incidence of some adverse events).

\section{Methods}

\section{Search strategy and selection criteria}

We searched Pubmed, Embase and Cochrane Library, from inception until June 2019 to identify systematic reviews, meta-analyses and pooled analyses of interventional studies investigating associations between AM and clinical outcomes. The search strategy as follows: (aliskiren OR direct renin inhibitor OR renin-angiotensin inhibition OR spp100 OR takturna) AND (systematic review OR meta-analysis OR pooled analysis). We also hand-searched all reference lists of the included studies to identify additional reviews of relevance. We used Endnote X9 to screen literatures. Two researchers screened the titles and abstracts independently, then reviewed the full text of selected articles and evaluated their eligibility. Any discrepancies were resolved with discussion or the arbitration of the third author.

\section{Inclusion and exclusion criteria}

We included English articles; The meta-analysis, systematic review or pooled analysis; meta-analyses that integrated the randomized controlled trials (RCT) which evaluated efficacy, tolerability or safety of AM. The latest edition was selected priority. Articles that had substantial data were included.

Observational studies, conference briefs, editorials and overview articles were excluded. We also excluded metaanalyses in which the intervening measures only included aliskiren combination therapy, or the primary outcome was not related to clinical efficacy, safety or tolerability. Meta-analyses that did not provide specific study data [number of incident events, number of study population, follow-up period, relative risks and $95 \%$ confidence intervals $(\mathrm{CI})]$ and in which the missing data was not retrievable from the original studies were also excluded.

\section{Data extraction}

Data extraction tables were established in Microsoft software. Two researchers extracted the data independently and discrepancies were resolved with consensus, if necessary, a third author would be involved. For each eligible article, extraction information as follows: the first author, year of publication, number of trials included, comparative drugs, follow-up period and the investigated clinical outcomes. We also calculated the study specific risk estimates [i.e., risk ratio (RR), odds ratio (OR), weighted mean difference (WMD), standard mean difference (SMD)] together with their 95\% CI and number of incident events and total events in each study.

If more than one meta-analysis compared aliskiren with the same drug evaluating the same outcome, we will incorporate all the original trials from those meta analyses, without including duplicates. All the available data were synthesized to get more comprehensive and objective result.

\section{Data analysis}

For each outcome, If the random model was not used, we will extracted data and estimated 95\% CI using random effects methods by ourselves. Then calculated $95 \%$ predication intervals (PI) for each random effect estimate, to represent the range in which the effect estimates of future studies will lie [12].

If possible, we would stratify the comparisons into several groups according to the dose, such as low group, low to high group, high group. In each group, the doses of aliskiren and control drug are comparable. We also stratified the comparisons according to the categories of contrast drugs. For example, we divided ARBs into three specific drugs: losartan, valsartan and irbesartan.

Between-study heterogeneity was quantified using the $I^{2}$ metric. $I^{2}$ ranges between 0 and $100 \%$ and quantifies the variability in effect estimates that is due to heterogeneity rather than sampling error [13]. Values 
exceeding $50 \%$ or $75 \%$ are considered to represent substantial or considerable heterogeneity.

Furthermore, if an estimate included at least 3 articles, we would reanalyse the estimate with Egger's asymmetry test, to detect and visualize the possible publication bias in the article. Revman and STATA 14.0 were used.

\section{Evaluation of quality of included studies}

We evaluated the quality of all included studies using the AMSTAR 2 tool, a comprehensive critical appraisal instrument that evaluated different aspects of reviews, to distinguish high quality ones [14].

\section{Patient and public involvement}

No patients will be involved in developing plans for project and implementation of the study. None of them will be asked to advise on interpretation of results. The results will be disseminated to the ordinary population through public presentations by the authors.

\section{Results}

\section{Eligible studies}

The literature search yielded 235 articles, of which 14 articles met our inclusion standard (see Fig. 1). Eleven are meta analyses or systematic reviews, only three are pooled analyses, all of them are the analyses of RCTs. The included articles were published from 2010 to 2019.

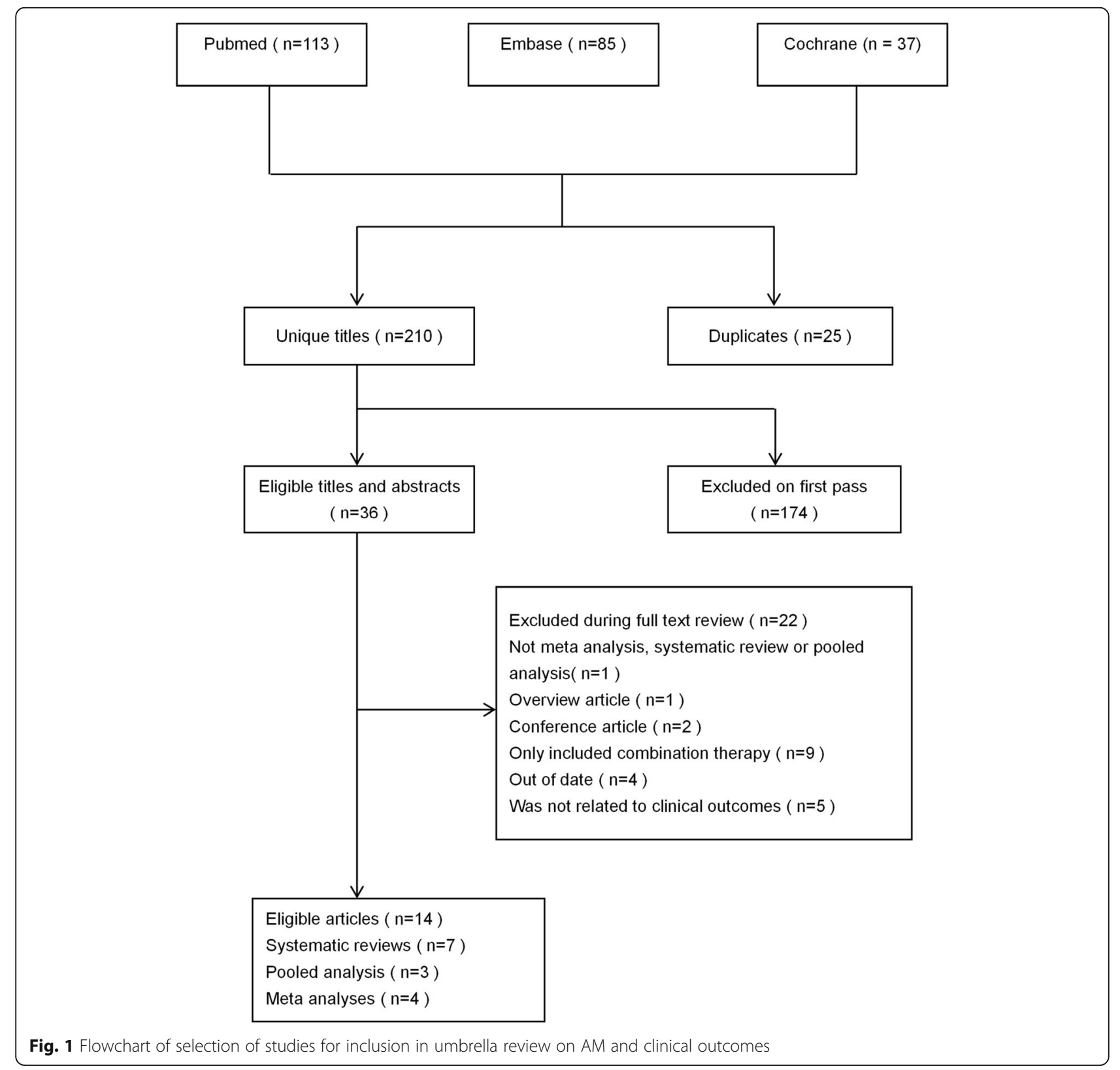


The 14 eligible articles contained a large number of meta-analyses and several unique outcomes. These meta-analyses are the comparisons between aliskiren and other pharmaceutical drugs, in aim of evaluating the association between AM and antihypertensive effects, the incidence of adverse events (for hypertension patients), cardiovascular outcomes (for HF patients) and renal effects (for different types of patients). More than one measurement index would be included for each outcome,.

\section{Antihypertensive effects}

To evaluate clinical value of AM in essential hypertension patients, we compared aliskiren with other antihypertensive drugs in four ways, including BP reduction, $\mathrm{BP}$ response rate, $\mathrm{BP}$ control rate, the incidence of adverse events.

\section{$B P$ reduction}

When comparing aliskiren to placebo, according to the dose of aliskiren used (75 mg, $150 \mathrm{mg}, 300 \mathrm{mg}, 600 \mathrm{mg}$ ), we stratified the comparisons into four groups $[8,15]$. Independent of the dose, aliskiren reduced BP to a greater degree. After using aliskiren for 8-26 weeks, both diastolic blood pressure (DBP) and systolic blood pressure (SBP) dropped significantly.

When comparing aliskiren to ARBs, we divided the comparisons into three groups: low dose group (aliskiren $150 \mathrm{mg}$ ), low to high dose group (aliskiren 150-300 mg), high dose group (aliskiren $300 \mathrm{mg}$ ) [16, 17]. In all three groups, the doses of aliskiren and ARBs were comparable. However, all the results showed that reductions from baseline to endpoint in both DBP and SBP did not differ between these two drugs.

When comparing aliskiren to ACEIs in the effects of $B P$ reduction, our study included two meta analyses [15, 18]. Aliskiren was slightly superior to ACEIs in reducing both DBP and SBP.

Aliskiren was inferior to amlodipine in reducing BP. Aliskiren and HCTZ showed no difference in BP reduction. Aliskiren was inferior to atenolol in reducing DBP, though two drugs showed no difference in SBP reduction.

When comparing aliskiren150mg to aliskiren $75 \mathrm{mg}$, aliskiren300mg to aliskiren150mg. With an increase of dosage, the effect of lowering DBP and SBP both significantly improved. However, according to the results, aliskiren $300 \mathrm{mg}$ and $600 \mathrm{mg}$ had similar effects in lowering BP [see Table 1 Reductions in mean sitting DBP (msDBP) and mean sitting DBP (msSBP)].

\section{$B P$ response rate}

$\mathrm{BP}$ response rate is defined as the percentage of patients with a mean DBP $<90 \mathrm{mmHg}$ and/or at least $10 \mathrm{mmHg}$ reduction from baseline [15]. When compared with placebo, aliskiren created statistically significant improvements on response rates in all doses. When compared with amlodipine and atenolol, aliskiren resulted in fewer patients receiving BP response. Aliskiren and ARBs, ACEIs, HCTZ showed comparable results in the proportion of patents received BP response (see Table 2 $\mathrm{BP}$ response rate and $\mathrm{BP}$ control rate).

\section{BP control rate}

$\mathrm{BP}$ control rate is defined as the percentage of patients with a mean $\mathrm{DBP}<90 \mathrm{mmHg}$ and mean $\mathrm{SBP}<140$ $\mathrm{mmHg}$ [15]. When compared with placebo, aliskiren resulted in a greater BP control rate in different doses, aside from aliskiren $75 \mathrm{mg}$. When compared with amlodipine, aliskiren resulted in a smaller BP control rate. Aliskiren and ARBs, ACEIs, HCTZ, atenolol showed comparable results in the proportion of patents received BP control (see Table 2).

\section{The incidence of adverse events}

When compared with placebo, according to the dose of aliskiren used $(75 \mathrm{mg}, 150 \mathrm{mg}, 300 \mathrm{mg}, 600 \mathrm{mg}$ ), we stratified the comparisons into four groups [8]. The results showed that aliskiren600mg resulted in more adverse events than placebo. Aliskiren $150 \mathrm{mg}$ and $600 \mathrm{mg}$ led to fewer withdrawals due to adverse events than placebo. Except for the above special cases, the incidence of total adverse events, severe adverse events and withdrawal due to adverse events are comparable between aliskiren and placebo.

When compared with ARBs, aliskiren showed no difference between two drugs in the number or extent of adverse events [16]. Then we divided ARBs into three specific drugs: losartan, valsartan and irbesartan, the results were unchanged.

When compared aliskiren with some active comparators $[15,19]$ (ACEIs, amlodipine, HCTZ or atenolol), the results showed no difference in adverse events or in withdrawals due to adverse events (see Table 3 The incidence of adverse events, serious adverse events and withdrawal due to adverse events).

In order to evaluate the paradoxical pressure rises after using aliskiren, we included a systematic review involving 4877 patients [20]. The results showed that when compared with placebo, increases in BP were less frequent in the aliskiren group. When compared with some active comparators (ARBs, ramipril or HCT), the incidence of BP increases with aliskiren was comparable (see Table 4 The incidence of paradoxical BP rises).

\section{Cardiovascular outcomes}

In order to evaluate the efficacy of aliskiren in HF patients, our study included a most recent article written by Luo $\mathrm{Y}$ and Chen Q [21]. The study compared 
Table 1 Reductions in mean sitting DBP (msDBP) and mean sitting DBP (msSBP)

\begin{tabular}{|c|c|c|c|c|c|c|c|c|c|}
\hline Outcome & $\begin{array}{l}\text { No. of } \\
\text { SRs }\end{array}$ & $\begin{array}{l}\text { No. of original } \\
\text { studies }\end{array}$ & $\begin{array}{l}\text { No. of cases/ } \\
\text { controls }\end{array}$ & $\begin{array}{l}\text { Follow-up } \\
\text { range (weeks) }\end{array}$ & Estimate $(95 \% \mathrm{Cl})$ & $P$-value & $95 \% \mathrm{PI}$ & $P^{2}\left(P^{*}\right)$ & $\begin{array}{l}\text { Egger's } \\
P \text { value }\end{array}$ \\
\hline \multicolumn{10}{|l|}{ msDBP } \\
\hline $\begin{array}{l}\text { aliskiren } 75 \mathrm{mg} \\
\text { vs placebo }\end{array}$ & 1 & 5 & $821 / 279$ & $8-13$ & $-2.05[-3.13,-0.96]$ & $<0.001$ & {$[-3.81,-0.28]$} & $0 \%(0.73)$ & 0.043 \\
\hline $\begin{array}{l}\text { aliskiren150mg } \\
\text { vs placebo }\end{array}$ & 1 & 12 & $2665 / 1118$ & $8-26$ & $-3.19[-4.02,-2.37]$ & $<0.001$ & {$[-5.57,-0.82]$} & $47 \%(0.04)$ & 0.798 \\
\hline $\begin{array}{l}\text { aliskiren300mg } \\
\text { vs placebo }\end{array}$ & 1 & 10 & $2193 / 808$ & $8-26$ & $-4.51[-5.27,-3.76]$ & $<0.001$ & {$[-5.97,-3.06]$} & $17 \%(0.29)$ & 0.359 \\
\hline $\begin{array}{l}\text { aliskiren600mg } \\
\text { vs placebo }\end{array}$ & 1 & 2 & $296 / 97$ & 8 & $-5.86[-7.73,-3.99]$ & $<0.0001$ & NA & $0 \%(0.57)$ & NA \\
\hline $\begin{array}{l}\text { aliskiren vs ARBs } \\
\text { (low dose) }\end{array}$ & 2 & 4 & $648 / 532$ & $4-8$ & $0.07[-0.94,1.09]$ & 0.89 & {$[-2.15,2.30]$} & $0 \%(0.48)$ & 0.12 \\
\hline $\begin{array}{l}\text { aliskiren vs ARBs } \\
\text { (low to high dose) }\end{array}$ & 2 & 5 & $923 / 944$ & $8-12$ & $-0.25[-2.32,1.82]$ & 0.81 & {$[-7.82,7.31]$} & $82 \%(0.0002)$ & 0.272 \\
\hline $\begin{array}{l}\text { aliskiren vs ARBs } \\
\text { (high dose) }\end{array}$ & 2 & 3 & $241 / 122$ & 8 & $-0.65[-2.52,1.23]$ & 0.5 & {$[-12.79,11.49]$} & $0 \%(0.89)$ & 0.293 \\
\hline aliskiren vs ACEls & 2 & 3 & 796/786 & $\geq 8$ & $-1.19[-1.99,-0.38]$ & 0.004 & {$[-6.42,4.05]$} & $0 \%(0.53)$ & 0.687 \\
\hline aliskiren vs amlodipine & 1 & 1 & 203/181 & 8 & $3.63[1.85,5.41]$ & $<0.0001$ & NA & NA & NA \\
\hline aliskiren vs HCTZ & 1 & 1 & 183/176 & 8 & $-0.9[-2.56,0.76]$ & 0.29 & NA & NA & NA \\
\hline aliskiren vs atenolol & 1 & 1 & $231 / 231$ & 12 & $2.4[0.74,4.06]$ & 0.004 & NA & NA & NA \\
\hline $\begin{array}{l}\text { Aliskiren150mg } \\
\text { vs } 75 \mathrm{mg}\end{array}$ & 1 & 5 & $830 / 824$ & $8-13$ & $-0.8[-1.58,-0.03]$ & 0.04 & {$[-2.06,0.04]$} & $0 \%(0.45)$ & 0.944 \\
\hline $\begin{array}{l}\text { aliskiren300mg } \\
\text { vs } 150 \mathrm{mg}\end{array}$ & 1 & 10 & $2193 / 2195$ & $8-26$ & $-1.75[-2.31,-1.20]$ & $<0.001$ & {$[-2.88,-0.63]$} & $20 \%(0.26)$ & 0.584 \\
\hline $\begin{array}{l}\text { aliskiren600mg } \\
\text { vs } 300 \mathrm{mg}\end{array}$ & 1 & 2 & $296 / 296$ & 8 & $-0.68[-2.03,0.67]$ & 0.449 & NA & $33 \%(0.22)$ & NA \\
\hline \multicolumn{10}{|l|}{ msSBP } \\
\hline $\begin{array}{l}\text { aliskiren } 75 \mathrm{mg} \\
\text { vs placebo }\end{array}$ & 1 & 5 & $821 / 279$ & $8-13$ & $-2.97[-4.76,-1.18]$ & 0.001 & {$[-5.88,-0.66]$} & $0 \%(0.57)$ & 0.253 \\
\hline $\begin{array}{l}\text { aliskiren } 150 \mathrm{mg} \\
\text { vs placebo }\end{array}$ & 1 & 12 & $2665 / 1121$ & $8-26$ & $-5.93[-6.94,-4.91]$ & $<0.001$ & {$[-7.93,-3.92]$} & $17 \%(0.28)$ & 0.674 \\
\hline $\begin{array}{l}\text { aliskiren300mg } \\
\text { vs placebo }\end{array}$ & 1 & 10 & 2199/810 & $8-26$ & $-7.91[-9.15,-6.67]$ & $<0.001$ & {$[-10.48,-5.33]$} & $22 \%(0.24)$ & 0.693 \\
\hline $\begin{array}{l}\text { aliskiren600mg } \\
\text { vs placebo }\end{array}$ & 1 & 2 & $296 / 97$ & 8 & $-11.35[-14.43,-8.27]$ & $<0.00001$ & NA & $0 \%(0.62)$ & NA \\
\hline $\begin{array}{l}\text { aliskiren vs ARBs } \\
\text { (low dose) }\end{array}$ & 2 & 4 & $648 / 532$ & $4-8$ & $1.25[-0.29,2.78]$ & 0.111 & {$[-2.57,5.06]$} & $6 \%(0.36)$ & 0.661 \\
\hline $\begin{array}{l}\text { aliskiren vs ARBs } \\
\text { (low to high dose) }\end{array}$ & 2 & 5 & $923 / 944$ & $8-12$ & $-1.19[-4.09,1.70]$ & 0.42 & {$[-11.46,9.08]$} & $77 \%(0.002)$ & 0.242 \\
\hline $\begin{array}{l}\text { aliskiren vs ARBs } \\
\text { (high dose) }\end{array}$ & 2 & 3 & $241 / 122$ & 8 & $0.24[-2.29,2.76]$ & 0.85 & {$[-16.10,16.57]$} & $0 \%(0.65)$ & 0.841 \\
\hline aliskiren vs ACEls & 2 & 4 & $1253 / 1230$ & $\geq 8$ & $-2.37[-3.48,-1.26]$ & $<0.0001$ & {$[-4.83,0.04]$} & $0 \%(0.59)$ & 0.123 \\
\hline aliskiren vs amlodipine & 1 & 1 & 203/181 & 8 & $5.67[2.86,8.48]$ & $<0.0001$ & NA & NA & NA \\
\hline aliskiren vs HCTZ & 1 & 1 & 183/176 & 8 & $-1.4[-4.04,1.24]$ & 0.3 & NA & NA & NA \\
\hline aliskiren vs atenolol & 1 & 1 & $231 / 231$ & 12 & $-0.08[-3.02,2.86]$ & 0.96 & NA & NA & NA \\
\hline $\begin{array}{l}\text { aliskiren150mg vs } \\
75 \mathrm{mg}\end{array}$ & 1 & 5 & $830 / 821$ & $8-13$ & $-1.89[-3.34,-0.43]$ & 0.011 & {$[-5.37,1.59]$} & $23 \%(0.27)$ & 0.872 \\
\hline $\begin{array}{l}\text { aliskiren300mg } \\
\text { vs } 150 \mathrm{mg}\end{array}$ & 1 & 10 & 2199/2195 & $8-26$ & $-2.57[-3.72,-1.42]$ & $<0.0001$ & {$[-5.87,0.73]$} & $52 \%(0.03)$ & 0.921 \\
\hline $\begin{array}{l}\text { aliskiren600mg } \\
\text { vs } 300 \text { mg }\end{array}$ & 1 & 2 & $296 / 296$ & 8 & $-0.61[-2.78,1.56]$ & 0.581 & NA & $0 \%(0.60)$ & NA \\
\hline
\end{tabular}

Notes: Type of metric: WMD (Weighted mean difference) Abbreviations: NA Not Accessible, SR Systematic review 
Table 2 BP response rate and BP control rate

\begin{tabular}{|c|c|c|c|c|c|c|c|c|c|}
\hline Outcome & No.of SRs & $\begin{array}{l}\text { No.of original } \\
\text { studies }\end{array}$ & $\begin{array}{l}\text { No.of cases/ } \\
\text { controls }\end{array}$ & $\begin{array}{l}\text { Follow-up } \\
\text { range (weeks) }\end{array}$ & $\begin{array}{l}\text { Estimate } \\
(95 \% \mathrm{Cl})\end{array}$ & $P$-value & $95 \% \mathrm{PI}$ & $P^{2}\left(\mathrm{P}^{*}\right)$ & $\begin{array}{l}\text { Egger's } \\
P \text { value } \\
\end{array}$ \\
\hline \multicolumn{10}{|l|}{ BP response rate } \\
\hline $\begin{array}{l}\text { aliskiren } 75 \mathrm{mg} \\
\text { vs placebo }\end{array}$ & 2 & 2 & $292 / 291$ & 8 & $1.41[1.03-1.94]$ & 0.03 & NA & $62 \%(0.11)$ & NA \\
\hline $\begin{array}{l}\text { aliskiren150mg } \\
\text { vs placebo }\end{array}$ & 2 & 3 & $460 / 451$ & 8 & $1.42[1.16-1.75]$ & 0.001 & {$[0.19-10.87]$} & $43 \%(0.17)$ & 0.008 \\
\hline $\begin{array}{l}\text { aliskiren300mg } \\
\text { vs placebo }\end{array}$ & 2 & 7 & $1443 / 1480$ & 8 & $1.65[1.46-1.85]$ & $<0.001$ & {$[1.17-2.35]$} & $49 \%(0.07)$ & 0.167 \\
\hline $\begin{array}{l}\text { aliskiren vs ARBs } \\
\text { (low dose) }\end{array}$ & 2 & 2 & $479 / 361$ & $4-8$ & $0.92[0.81-1.04]$ & 0.16 & NA & $0 \%(0.89)$ & NA \\
\hline $\begin{array}{l}\text { aliskiren vs ARBs } \\
\text { (low to high dose) }\end{array}$ & 2 & 2 & $543 / 570$ & $8-12$ & 0.99 [0.90-1.09] & 0.88 & NA & $0 \%(0.51)$ & NA \\
\hline $\begin{array}{l}\text { aliskiren vs ARBs } \\
\text { (high dose) }\end{array}$ & 1 & 1 & $175 / 60$ & 8 & $1.07[0.86-1.33]$ & 0.52 & NA & NA & NA \\
\hline aliskiren vs ACEls & 1 & 1 & $414 / 418$ & $8-26$ & $1.10[0.99-1.24]$ & 0.09 & NA & NA & NA \\
\hline aliskiren vs amlodipine & 1 & 2 & $513 / 492$ & $8-32$ & $0.77[0.69-0.85]$ & $<0.00001$ & NA & $0 \%(0.37)$ & NA \\
\hline aliskiren vs HCTZ & 1 & 1 & $180 / 173$ & 8 & $1.08[0.92-1.28]$ & 0.34 & NA & NA & NA \\
\hline aliskiren vs atenolol & 1 & 1 & $230 / 230$ & 12 & $0.84[0.74-0.95]$ & 0.007 & NA & NA & NA \\
\hline \multicolumn{10}{|l|}{ BP control rate } \\
\hline $\begin{array}{l}\text { aliskiren } 75 \mathrm{mg} \\
\text { vs placebo }\end{array}$ & 2 & 1 & $177 / 176$ & 8 & $1.30[0.95-1.77]$ & 0.1 & NA & NA & NA \\
\hline $\begin{array}{l}\text { aliskiren150mg } \\
\text { vs placebo }\end{array}$ & 2 & 3 & $475 / 666$ & 8 & $1.51[1.06-2.16]$ & 0.02 & {$[0.03-76.62]$} & $62 \%(0.07)$ & 0.084 \\
\hline $\begin{array}{l}\text { aliskiren300mg } \\
\text { vs placebo }\end{array}$ & 2 & 6 & $1276 / 1288$ & 8 & $1.62[1.10-2.38]$ & 0.01 & {$[0.41-6.36]$} & $90 \%(<0.00001)$ & 0.741 \\
\hline $\begin{array}{l}\text { aliskiren vs ARBs } \\
\text { (low to high dose) }\end{array}$ & 2 & 5 & 919/921 & $8-12$ & $1.05[0.89-1.23]$ & 0.57 & {$[0.68-1.62]$} & $37 \%(0.18)$ & 0.357 \\
\hline $\begin{array}{l}\text { aliskiren vs ARBs } \\
\text { (high dose) }\end{array}$ & 1 & 1 & $175 / 60$ & 8 & $1.01[0.72-1.43]$ & 0.93 & NA & NA & NA \\
\hline aliskiren vs ACEls & 1 & 1 & $414 / 418$ & $8-26$ & $1.12[0.96-1.30]$ & 0.15 & NA & NA & NA \\
\hline aliskiren vs amlodipine & 1 & 1 & 201/179 & 8 & $0.72[0.57-0.91]$ & 0.006 & NA & NA & NA \\
\hline aliskiren vs HCTZ & 1 & 1 & $180 / 173$ & 8 & 1.24 [0.97-1.59] & 0.09 & NA & NA & NA \\
\hline aliskiren vs atenolol & 1 & 1 & $230 / 230$ & 12 & $0.86[0.68-1.08]$ & 0.18 & NA & NA & NA \\
\hline
\end{tabular}

Notes: Type of metric for comparisons: RR (Risk ratio)

Abbreviations: NA Not Accessible, NR Not Report, SA Systematic review

aliskiren with placebo in the changes in some biological markers, aliskiren and placebo did not differ in BNP levels or mortality. However, aliskiren led to a significantly decreased NT-proBNP level (SMD -0.12, -0.21 to -0.03$)$. Furthermore, aliskiren led to a significant reduced PRA (SMD $-0.66,-0.89$ to -0.44 ) and increased PRC (SMD 0.52, 0.30 to 0.75). No significant difference between the two drugs in aldosterone levels either.

In a separate article, the authors compared aliskiren with placebo in incidence of related cardiovascular events like myocardial infarction and stroke [22]. Results showed no difference between these two drugs. However, the study included only one article and 613 patients, results need to be further discussed. Both articles evaluated the association betweeen AM and all- cause mortality. The results showed that aliskiren did not affect the frequency of death in HF patients.

In another included article [23], the authors evaluated combined cardiovascular disease (CVD) mortality and HF hospitalization, aliskiren and enalapril showed no difference in the outcomes (see Table 5 The efficacy of aliskiren in heart failure).

\section{Renal effects}

To evaluate renoprotective effects of aliskiren, our study included several articles. For essential hypertension patients, our study included two studies compared aliskiren with placebo, ARBs and HCT in changes of some closely related outcome indicators [20,24]. All the results failed to reach statistical significance, aliskiren showed no difference three drugs. Hyperkalemia is also an important 
Table 3 The incidence of adverse events, serious adverse events and withdrawal due to adverse events

\begin{tabular}{|c|c|c|c|c|c|c|c|c|c|}
\hline Outcome & No.of SRs & $\begin{array}{l}\text { No.of original } \\
\text { studies }\end{array}$ & $\begin{array}{l}\text { No.of cases/ } \\
\text { controls }\end{array}$ & $\begin{array}{l}\text { Follow-up } \\
\text { range (weeks) }\end{array}$ & $\begin{array}{l}\text { Estimate } \\
(95 \% \mathrm{Cl})\end{array}$ & $P$-value & $95 \% \mathrm{PI}$ & $P^{2}\left(P^{*}\right)$ & $\begin{array}{l}\text { Egger's } \\
P \text { value }\end{array}$ \\
\hline \multicolumn{10}{|l|}{ Adverse events } \\
\hline aliskiren 75 mg vs placebo & 1 & 5 & $742 / 740$ & $8-13$ & $0.96[0.84-1.08]$ & 0.48 & {$[0.78-1.17]$} & $0 \%(0.56)$ & 0.853 \\
\hline aliskiren150mg vs placebo & 1 & 10 & $1785 / 1639$ & $8-26$ & 0.99 [0.90-1.08] & 0.77 & [0.89-1.10] & $0 \%(0.65)$ & 0.43 \\
\hline aliskiren300mg vs placebo & 1 & 9 & $1461 / 1478$ & $8-26$ & $1.02[0.93-1.12]$ & 0.68 & {$[0.91-1.15]$} & $0 \%(0.47)$ & 0.876 \\
\hline aliskiren600mg vs placebo & 1 & 3 & $499 / 494$ & 8 & $1.24[1.07-1.43]$ & 0.004 & {$[0.49-3.13]$} & $0 \%(0.88)$ & 0.083 \\
\hline aliskiren vs ARBs & 1 & $N R$ & $N R$ & $4-8$ & 0.98 [0.89-1.08] & 0.68 & $N R$ & $N R$ & $N R$ \\
\hline aliskiren vs lorsartan & 1 & $N R$ & NR & $4-8$ & $1.03[0.79-1.35]$ & 0.83 & $N R$ & NR & NR \\
\hline aliskiren vs valsartan & 1 & NR & NR & 8 & $0.92[0.81-1.05]$ & 0.2 & $N R$ & $N R$ & $N R$ \\
\hline aliskiren vs irbesartan & 1 & $N R$ & $N R$ & $7-8$ & $1[0.81-1.23]$ & 0.99 & $N R$ & $N R$ & $N R$ \\
\hline aliskiren vs ACEls & 1 & 2 & $514 / 508$ & $8-26$ & $1[0.89-1.11]$ & 0.93 & NA & $0 \%$ & NA \\
\hline aliskiren vs Amlodipine & 1 & 2 & $513 / 492$ & $8-32$ & $0.99[0.81-1.11]$ & 0.92 & NA & $37 \%$ & NA \\
\hline aliskiren vs HCTZ & 1 & 1 & 183/176 & 8 & $0.95[0.74-1.22]$ & 0.68 & NA & NA & NA \\
\hline aliskiren vs atenolol & 1 & 1 & $231 / 231$ & 12 & $0.88[0.72-1.08]$ & 0.23 & NA & NA & NA \\
\hline \multicolumn{10}{|l|}{ Serious adverse events } \\
\hline aliskiren vs placebo & 1 & 8 & $3633 / 1683$ & $8-26$ & $0.75[0.27-2.05]$ & 0.57 & {$[0.07-7.71]$} & $32 \%(0.17)$ & 0.811 \\
\hline aliskiren vs ARBS & 1 & $N R$ & $N R$ & $4-8$ & $0.72[0.36-1.46]$ & 0.36 & $N R$ & $N R$ & $N R$ \\
\hline aliskiren vs lorsartan & 1 & NR & NR & $4-8$ & $0.33[0.01-8.18]$ & 0.5 & NR & $N R$ & $N R$ \\
\hline aliskiren vs valsartan & 1 & $N R$ & $N R$ & 8 & $0.63[0.09-4.43]$ & 0.65 & $N R$ & NR & NR \\
\hline aliskiren vs irbesartan & 1 & $N R$ & NR & $7-8$ & $0.55[0.18-1.67]$ & 0.29 & NR & NR & NR \\
\hline \multicolumn{10}{|c|}{ Withdrawal due to adverse events } \\
\hline aliskiren 75 mg vs placebo & 1 & 5 & $821 / 817$ & $8-13$ & $0.69[0.37-1.29]$ & 0.24 & {$[0.25-1.90]$} & $0 \%(0.52)$ & 0.008 \\
\hline aliskiren150mg vs placebo & 1 & 7 & $1161 / 1154$ & $8-26$ & $0.37[0.16-0.84]$ & 0.02 & {$[0.05-2.75]$} & $38 \%(0.14)$ & 0.065 \\
\hline aliskiren300mg vs placebo & 1 & 6 & $984 / 1001$ & $8-26$ & $0.60[0.34-1.06]$ & 0.08 & {$[0.21-1.74]$} & $12 \%(0.34)$ & 0.365 \\
\hline aliskiren600mg vs placebo & 1 & 1 & $166 / 165$ & 8 & $0.14[0.03-0.62]$ & 0.009 & NA & NA & NA \\
\hline aliskiren vs ARBs & 1 & NR & NR & $4-8$ & $0.82[0.54-1.25]$ & 0.35 & NR & $N R$ & NR \\
\hline aliskiren vs lorsartan & 1 & $N R$ & NR & $4-8$ & $0.76[0.28-2.08]$ & 0.6 & $N R$ & $N R$ & $N R$ \\
\hline aliskiren vs valsartan & 1 & NR & NR & 8 & $0.89[0.50-1.58]$ & 0.69 & NR & $N R$ & $N R$ \\
\hline aliskiren vs irbesartan & 1 & $N R$ & NR & $7-8$ & $0.73[0.33-1.61]$ & 0.43 & NR & NR & NR \\
\hline aliskiren vs ACEls & 2 & 3 & $1145 / 952$ & $8-36$ & $0.76[0.45-1.28]$ & 0.3 & {$[0.00-149.77]$} & $50 \%(0.13)$ & 0.953 \\
\hline aliskiren vs Amlodipine & 1 & 2 & $513 / 492$ & $8-32$ & $0.42[0.08-2.30]$ & 0.32 & NA & $65 \%$ & NA \\
\hline aliskiren vs HCTZ & 1 & 1 & 183/176 & 8 & $1.92[0.59-6.27]$ & 0.28 & NA & NA & NA \\
\hline aliskiren vs atenolol & 1 & 1 & $231 / 231$ & 12 & $0.6[0.22-1.62]$ & 0.31 & NA & NA & NA \\
\hline
\end{tabular}

Notes: Type of metric for comparisons: RR (Risk ratio)

Abbreviations: NA Not Accessible, NR Not Report, SA Systematic review

indication for evaluating renal effects. The occurrence of hyperkalemia is comparable between aliskiren and placebo, ARBs or HCT.

In different type of patients, our study included a meta analysis [25] comparing aliskiren to placebo in increases in serum creatinine $(\mathrm{sCr})$ or decreases in estimated glomerular filtration rate (eGFR). The results showed aliskiren had renoprotective influence and led to fewer renal impairment events, though this did not reach statistical significance (RR $0.97,0.81$ to 1.16 ) [21] (see Table 6).
For patients who are suffered from hypertension and diabetes and/or nephropathy or albuminuria at the same time, our study included two reviews [25, 26]: Results showed that in non-diabetic chronic kidney disease patients, 300 mg aliskiren reduced Urinary Protein Excretion Rate (UPER) better than placebo $(p<0.05)$ and $10 \mathrm{mg}$ perindopril $[\mathrm{p}=$ no significance (NS)]. In IgA nephropathy patients, $300 \mathrm{mg}$ aliskiren also reduced Urinary Protein Creatinine Rate (UPCR). In T2DM and hypertension and albuminuria patients, $300 \mathrm{mg}$ aliskiren reduced Urinary Albumin Excretion Rate (UAER) better than placebo $(p<0.001)$, worse 
Table 4 The incidence of paradoxical BP rises

\begin{tabular}{|c|c|c|c|c|c|c|}
\hline Outcome & No. of SRs & No. of original studies & No. of cases/controls & Follow-up range (weeks) & Estimate $(95 \% \mathrm{Cl})$ & $P$-value \\
\hline \multicolumn{7}{|c|}{ Paradoxical blood pressure rise: $\Delta \mathrm{msDBP}>5 \mathrm{mmHg}$} \\
\hline aliskiren vs placebo & 1 & 4 & $2110 / 902$ & 8 & $0.27[0.20-0.37]$ & $<0.00001$ \\
\hline aliskiren vs ARBs & 1 & 4 & $2110 / 701$ & $4-9$ & $0.86[0.55-1.36]$ & 0.53 \\
\hline aliskiren vs ramipril & 1 & 2 & $2110 / 617$ & $9-26$ & $1.19[0.69-2.04]$ & 0.53 \\
\hline aliskiren vs HCT & 1 & 1 & $2110 / 547$ & 52 & $1.20[0.68-2.13]$ & 0.52 \\
\hline \multicolumn{7}{|l|}{$\triangle \mathrm{msDBP}>10 \mathrm{mmHg}$} \\
\hline aliskiren vs placebo & 1 & 4 & $2110 / 902$ & 8 & $0.27[0.15-0.48]$ & $<0.00001$ \\
\hline aliskiren vs ARBs & 1 & 4 & $2110 / 701$ & $4-9$ & $0.57[0.27-1.20]$ & 0.14 \\
\hline aliskiren vs ramipril & 1 & 2 & $2110 / 617$ & $9-26$ & $0.93[0.37-2.31]$ & 0.87 \\
\hline aliskiren vs HCT & 1 & 1 & $2110 / 547$ & 52 & 0.70 [0.30-1.67] & 0.42 \\
\hline \multicolumn{7}{|l|}{$\Delta \mathrm{msSBP}>10 \mathrm{mmHg}$} \\
\hline aliskiren vs placebo & 1 & 4 & $2110 / 902$ & 8 & $0.31[0.24-0.41]$ & $<0.00001$ \\
\hline aliskiren vs ARBs & 1 & 4 & $2110 / 701$ & $4-9$ & 0.97 [0.64-1.48] & 0.9 \\
\hline aliskiren vs ramipril & 1 & 2 & $2110 / 617$ & $9-26$ & $0.69[0.47-1.01]$ & 0.05 \\
\hline aliskiren vs HCT & 1 & 1 & $2110 / 547$ & 52 & 0.89 [0.57-1.38] & 0.59 \\
\hline \multicolumn{7}{|l|}{$\Delta \mathrm{msSBP}>20 \mathrm{mmHg}$} \\
\hline aliskiren vs placebo & 1 & 4 & $2110 / 902$ & 8 & $0.40[0.24-0.67]$ & 0.0005 \\
\hline aliskiren vs ARBs & 1 & 4 & $2110 / 701$ & $4-9$ & $1.50[0.62-3.61]$ & 0.37 \\
\hline aliskiren vs ramipril & 1 & 2 & $2110 / 617$ & $9-26$ & 0.66 [0.34-1.29] & 0.22 \\
\hline aliskiren vs HCT & 1 & 1 & $2110 / 547$ & 52 & 0.87 [0.40-1.92] & 0.74 \\
\hline
\end{tabular}

Notes: Type of metric for comparsions: RR (Risk ratio)

Abbreviations: SA Systematic review

Table $\mathbf{5}$ The efficacy of aliskiren in heart failure

\begin{tabular}{|c|c|c|c|c|c|c|c|c|c|c|}
\hline Outcome & $\begin{array}{l}\text { No. of } \\
\text { SRs }\end{array}$ & $\begin{array}{l}\text { No. of } \\
\text { original } \\
\text { studies }\end{array}$ & $\begin{array}{l}\text { No. of cases/ } \\
\text { controls }\end{array}$ & $\begin{array}{l}\text { Follow-up } \\
\text { range (weeks) }\end{array}$ & $\begin{array}{l}\text { Type of } \\
\text { metric }\end{array}$ & $\begin{array}{l}\text { Estimate } \\
(95 \% \mathrm{Cl})\end{array}$ & $P$-value & 95\%PI & $I^{2}\left(P^{*}\right)$ & $\begin{array}{l}\text { Egger's } \\
P \text { value }\end{array}$ \\
\hline \multicolumn{11}{|l|}{ aliskiren vs placebo } \\
\hline NT-proBNP levels & 1 & 3 & $975 / 956$ & $12-48$ & SMD & $-0.12[-0.21,-0.03]$ & 0.01 & {$[-0.70,0.46]$} & $0 \%(0.57)$ & 0.814 \\
\hline BNP levels & 1 & 2 & $151 / 143$ & $6-12$ & SMD & $-0.08[-0.31,0.15]$ & 0.49 & NA & $0 \%(0.76)$ & NA \\
\hline $\begin{array}{l}\text { Plasma renin } \\
\text { activity }\end{array}$ & 1 & 3 & $176 / 157$ & $12-48$ & SMD & $-0.66[-0.89,-0.44]$ & $<0.0001$ & {$[-2.11,0.77]$} & $0 \%(0.85)$ & 0.648 \\
\hline $\begin{array}{l}\text { Plasma renin } \\
\text { concentration }\end{array}$ & 1 & 2 & $167 / 149$ & $6-26$ & SMD & $0.52[0.30,0.75]$ & $<0.0001$ & NA & $0 \%(0.72)$ & NA \\
\hline Aldosterone level & 1 & 2 & $151 / 143$ & $6-12$ & SMD & $-0.09[-0.32,0.14]$ & 0.44 & NA & $0 \%(0.55)$ & NA \\
\hline Mortality & 2 & 3 & $1255 / 1250$ & $\geq 12$ & $\mathrm{RR}$ & $0.76[0.32-1.80]$ & 0.53 & {$[0.00,3273.53]$} & $24 \%(0.27)$ & 0.498 \\
\hline Myocardial infarction & 1 & 1 & $305 / 308$ & 104 & $\mathrm{RR}$ & $0.13[0.02-1.00]$ & 0.05 & NA & NA & NA \\
\hline Stroke & 1 & 1 & $305 / 308$ & 104 & $\mathrm{RR}$ & $0.25[0.03-2.25]$ & 0.22 & NA & NA & NA \\
\hline \multicolumn{11}{|l|}{ aliskiren vs enalapril } \\
\hline $\begin{array}{l}\text { Combined } \\
\text { cardiovascular } \\
\text { mortality and } \\
\text { hospitalisation }\end{array}$ & 1 & 1 & $2340 / 2336$ & 36 & $\mathrm{RR}$ & 0.98 [0.90-1.06] & 0.57 & NA & NA & NA \\
\hline
\end{tabular}


Table 6 Aliskren monotherapy and renal effects in simple hypertension patients and different types of patients

\begin{tabular}{|c|c|c|c|c|c|c|}
\hline Outcomes & No. of SRs & No. of original studies & No. of cases/controls & Follow-up range (weeks) & Estimate $(95 \% \mathrm{Cl})$ & $P$-value \\
\hline \multicolumn{7}{|l|}{ changes of sCr or eGFR } \\
\hline aliskiren vs placebo & 1 & 3 & $6812 / 5448$ & NA & $0.97[0.81-1.16]$ & 0.73 \\
\hline \multicolumn{7}{|l|}{$\mathrm{BUN}>40 \mathrm{mg} / \mathrm{dL}$} \\
\hline aliskiren vs placebo & 1 & NA & $1508 / 753$ & 8 & $1.50[0.06-36.75]$ & 0.8 \\
\hline aliskiren vs ARBs & 1 & NA & $4579 / 1223$ & $8-52$ & $1.16[0.33-4.06]$ & 0.82 \\
\hline aliskiren vs HCT & 1 & NA & $4579 / 1113$ & $8-52$ & $0.79[0.26-2.42]$ & 0.68 \\
\hline \multicolumn{7}{|c|}{ Creatinine level > $2.0 \mathrm{mg} / \mathrm{dL}$} \\
\hline aliskiren vs placebo & 1 & NA & $1508 / 753$ & 8 & $1.50[0.06-36.75]$ & 0.8 \\
\hline aliskiren vs ARBs & 1 & NA & $4579 / 1223$ & $8-52$ & $0.71[0.19-2.68]$ & 0.62 \\
\hline aliskiren vs HCT & 1 & NA & $4579 / 1113$ & $8-52$ & $4.13[0.24-71.59]$ & 0.33 \\
\hline \multicolumn{7}{|c|}{$\mathrm{eGFR}<30 \mathrm{~mL} / \mathrm{min} / 1.73 \mathrm{~m} 2$} \\
\hline aliskiren vs ARBs & 1 & NA & $4579 / 1223$ & $8-52$ & $0.53[0.13-2.13]$ & 0.37 \\
\hline aliskiren vs HCT & 1 & NA & $4579 / 1113$ & $8-52$ & 3.16 [0.18-56.09] & 0.43 \\
\hline \multicolumn{7}{|l|}{ Hyperkalaemia } \\
\hline aliskiren vs placebo & 1 & NA & $1405 / 752$ & 8 & $1.40[0.51-3.87]$ & 0.52 \\
\hline aliskiren vs ARBs & 1 & NA & NA & $4-8$ & $0.93[0.51-1.70]$ & 0.82 \\
\hline aliskiren vs HCT & 1 & NA & $5450 / 1113$ & NA & 0.87 [0.62-1.24] & 0.43 \\
\hline
\end{tabular}

Notes: Type of metric for comparsions: RR (Risk ratio)

Abbreviations: NA Not Accessible, SA Systematic review

than $300 \mathrm{mg}$ irbesartan ( $p=\mathrm{NS}) .600 \mathrm{mg}$ aliskiren also performed better than the dose of $300 \mathrm{mg}(p=\mathrm{NS})$ and 150 mg $(p<0.05)$ (see Table 7).

\section{Prediction intervals and heterogeneity between studies} We calculated 95\%PI, the null value was excluded in only 21 meta analyses. Twenty-fivemeta-analyses showed no heterogeneity $\left(\mathrm{I}^{2}=0\right)$ for several outcomes. Substantial heterogeneity $\left(50 \% \leq \mathrm{I}^{2} \leq 75 \%\right)$ was present in 6 meta analyses addressing clinical outcomes. Considerable heterogeneity $\left(\mathrm{I}^{2}>75 \%\right)$ was present in only three meta-analyses in three outcomes (changes of SBP, DBP when comparing aliskiren150-300 mg with low to high ARBs, BP response rate when comparing aliskiren300mg with placebo).

\section{Publication bias of included studies}

We performed Egger's regression test in only 36 meta analyses in our reanalysis, because the remaining

Table 7 aliskren monotherapy and renal effects for patients who are suffered from hypertension and diabetes and/or nephropathy or albuminuria at the same time

\begin{tabular}{|c|c|c|c|c|c|}
\hline Author & Indication & Total Sample Size & Follow up & Drug Comparison & Primary Outcome - Results \\
\hline \multirow[t]{4}{*}{$\begin{array}{l}\text { Evangelos C. Rizos, } \\
\text { Aris P. Agouridis and } \\
\text { Moses S.Elisaf }\end{array}$} & $\begin{array}{l}\text { non-diabetic chronic } \\
\text { kidney disease }\end{array}$ & 14 & 42 & $\begin{array}{l}\text { placebo vs. aliskiren } 300 \text { mg } \\
\text { vs. perindopril } 10 \mathrm{mg}\end{array}$ & $\begin{array}{l}\text { UPER: }-36 \% \text { for aliskiren } \\
\text { and }-25 \% \text { for perindopril } \\
10 \mathrm{mg} \\
\text { Both treatments vs. placebo: } \\
p<0.05\end{array}$ \\
\hline & IgA nephropathy & 22 & 36 & placebo vs. aliskiren 300 mg & Reduction in UPCR (g/g) \\
\hline & $\begin{array}{l}\text { T2DM and hypertension } \\
\text { and albuminuria }\end{array}$ & 26 (crossover design) & $4 \times 2$-months & $\begin{array}{l}\text { Placebo vs. aliskiren300mg } \\
\text { vs. irbesartan } 300 \text { mg }\end{array}$ & $\begin{array}{l}\text { UAER: }-48 \% \text { for aliskiren } \\
\text { and }-51 \% \text { for irbesartan } \\
\text { Both treatments vs. placebo: } \\
p<0.001\end{array}$ \\
\hline & $\begin{array}{l}\text { T2DM and hypertension } \\
\text { and albuminuria }\end{array}$ & 26 (crossover design) & $4 \times 2$-months & $\begin{array}{l}150 \text { or } 300 \text { or } 600 \mathrm{mg} \\
\text { aliskiren vs. placebo }\end{array}$ & $\begin{array}{l}\text { UAER: }-52 \% \text { for } 600 \mathrm{mg},-48 \% \\
\text { for } 300 \mathrm{mg} \text {, } \\
-36 \% \text { for } 150 \mathrm{mg}(600 \mathrm{mg} \text { vs. } \\
150 \mathrm{mg}: p<0.05,600 \mathrm{mg} \text { vs. } \\
300 \mathrm{mg}: p=\mathrm{NS} \text { ) } \\
\text { All treatments vs. placebo: } \\
p<0.001\end{array}$ \\
\hline
\end{tabular}


analyses contained insufficient numbers of studies. In those we analysed, only two had statistical evidence of publication bias $(P<0.1)$. The two are aliskiren $150 \mathrm{mg}$ versus placebo in BP response rate $(P=$ 0.008 ), aliskiren $75 \mathrm{mg}$ versus placebo in withdrawal due to adverse events $(P=0.008)$. For those that can be calculated, publication bias of meta analyses included in our study were not existed.

\section{AMSTAR 2 classification of included studies}

When evaluating the important items, most of the studies used a comprehensive literature search strategy (10/14), justified for excluding individual studies (11/14), used a satisfactory technique for evaluating the risk of bias (RoB) in individual studies (9/14), gave thoughts to RoB of original trials when interpreting the results in the review (8/ 14). All of the included reviews stated explicitly that the review methods were established prior to the conduct and used appropriate methods to combine the results. But few of the studies considered the possibility of publication bias and discussed its likely impact (4/14).

When we examined the study as a whole, however, most of our included reviews had one or more defects in important items. According to the appraisal standard, one review has a critical flaw with or without noncritical weaknesses is considered low, one review has more than one critical flaw with or without non-critical weaknesses is considered critically low [14]. That explains why our included studies are mostly not of high quality. Overall, only three of included studies are rated high, and one is rated moderate, the other ten remaining studies are rated low or critically low (see Table 8 AMSTAR 2 classification of included studies).

\section{Discussion}

\section{Main findings and possible explanations}

Aliskiren supplement is commonly considered to be beneficial in the treatment of hypertension, HF and renal dysfunction. Supplement of aliskiren has been the subject of numerous meta-analyses on a varied range of outcomes. We performed this umbrella review to bring the existing evidence together and draw conclusions for the overall effects of AM on clinical outcomes. We identified a large number of meta analyses, systematic reviews and pooled analyses of RCTs with several important distinctive outcomes.

Positive effects of AM in the treatment of hypertension were supported by the findings in our study. Compared with placebo, aliskiren led to a greater degree in lowering $\mathrm{BP}$, a higher rate of $\mathrm{BP}$ response and $\mathrm{BP}$ control. Compared with ACEIs, It showed a good superiority in BP reduction and produced similar effects as ARBs and HCTZ on three efficacy outcomes.
Aliskiren also showed a good performance in terms of safety and tolerability. The incidence of adverse events, severe adverse events and withdrawal due to adverse events were mostly comparable between aliskiren and placebo or other comparator drugs. The incidence of some specific adverse events, including dizziness, fatigue, nausea, edema, bronchitis, infection, nasopharyngitis and back pain are comparable between aliskiren and placebo, ARBs or HCT [20, 24]. A single meta analysis showed that aliskiren had a larger chance of developing diarrhea than placebo, while only in the high dose group (aliskiren $=600 \mathrm{mg}$ ) [8]. However, headache was reported less frequently with aliskiren $150 \mathrm{mg}$ and $300 \mathrm{mg}$ than with placebo [20]. Hypotension was reported less frequently with AM than with thiazide diuretic monotherapy [24]. Furthermore, a meta analysis including two original studies showed that compared with rampril (a kind of ACEI drug), aliskiren reduced the rate of cough by $67 \%$ (RR0.33, 0.22 to 0.49 ) [19].

The antihypertensive effects of aliskiren may be explained by its association with a decreased generation of Ang I, as it blocks generation of Ang I from angiotensinogen, by inhibiting the active enzymatic site of renin. Furthermore, aliskiren can inhibit renin activity and block RAAS cascade at its primary steps, which provides an appropriate means of RAAS inhibition [7]. However, aliskiren should not be taken at a high dose, aliskiren $600 \mathrm{mg}$ resulted in more frequent and severe adverse events. Aliskiren $300 \mathrm{mg}$ is the most optimal because it presented significant effects of lowering BP and was safer. In a word, if used properly, aliskiren would be a trustworthy drug for essential hypertension patients.

In HF patients, results showed aliskiren significantly decreased NT-proBNP and PRA levels, increase PRC levels, but had no important influence on aldosterone and BNP levels. NT-proBNP, BNP, PRA, PRC, aldosterone are all biological markers connected tightly with heart failure. Of them, NT- pro BNP and BNP levels are particularly important, and can be used to evaluate the severity of disease [27]. However, when evaluating cardiovascular outcomes in HF patients, our results showed that compared with placebo, aliskiren showed no obvious effects on death rate, the incidence of myocardial infarction and stroke. Compared with enalapril, aliskiren showed no effects on combined CVD mortality and HF hospitalization. The outcomes suggested aliskiren therapy may have good results on some markers, but it would not reduce risk of cardiovascular outcomes, HF hospitalization or total mortality.

Similarly, according to another report, aliskiren led to a significant and sustained reduction in natriuretic peptide levels, but it did not reduce mortality or rehospitalization rate [28]. The explanation may be that there exists an unknown upper limit for the benefits of 


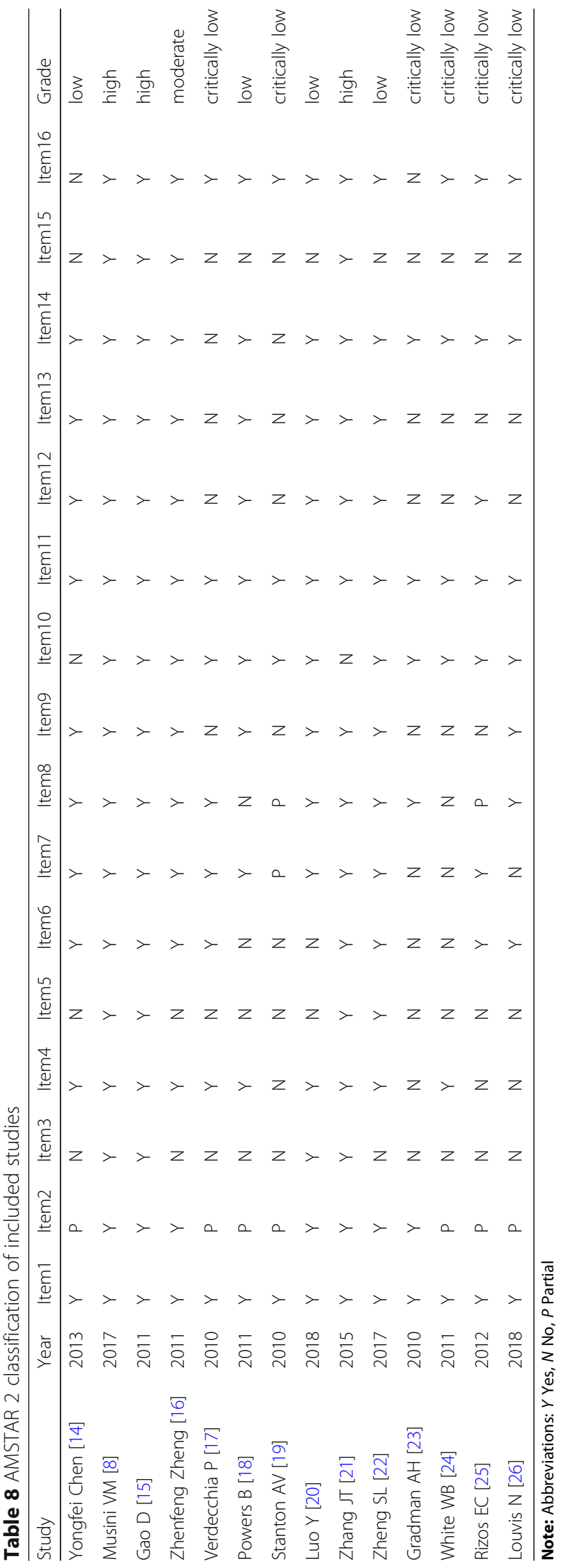


RAAS blockade, and over inhibition of it may cause malfunction [29]. Aliskiren, either used alone or in combination with standard medical therapies, is associated with more adverse events, including hyperkalaemia, renal dysfunction and hypotension [30]: These negative events would play a crucial role in offsetting any beneficial effects of aliskiren treatment on HF progression (with or without diabetes), as a result, patients' major cardiovascular outcomes would not be altered [31].

However, since aliskiren has the possibility to increase the risk of hypotension and hyperkalaemia, it may be expected to apply for HF patients who are suffered from hypertension or hypokalaemia at the same time [32]. Moreover, a small-scale published RCT has initially shown that aliskiren lowered arterial stiffness and left ventricular diastolic function in elderly hypertensive patients during a follow-up of 6 months [33]. The study showed that aliskiren can be indicated for HF with preserved ejection fraction patients. In summary, AM can only be clinically useful for a particular group of $\mathrm{HF}$ patients.

In terms of renal effects, for essential hypertension patients, the occurrence of hyperkalemia, blood urea nitrogen $(\mathrm{BUN})>40 \mathrm{mg} / \mathrm{dL}, \mathrm{sCr}>2.0 \mathrm{mg} / \mathrm{dL}$ and eGFR $<30$ $\mathrm{mL} / \mathrm{min} / 1.73 \mathrm{~m}^{2}$ are comparable between aliskiren and placebo, ARBs or HCT. For different types of patients, composite changes of $\mathrm{sCr}$ or eGFR are comparable between aliskiren and matched drugs. For patients who are suffered from hypertension and diabetes and/or nephropathy or albuminuria at the same time, our study showed that aliskiren had good performance in changes of surrogate markers. However, as our study included few RCTs and limited participants, examined no hard outcomes such as mortality, major cardiovascular or renal outcomes, the durations of our included trials were short, the results are not convincing.

The little influence of aliskiren effects on renal progression may be explained by its bilateral effects. On the one hand, hypertension can cause glomerular and tubular destruction in the kidneys, accelerating the development of kidney diseases. The decrease of BP has been reported to inhibit the progression of renal failure [34]. Thus, as an effective antihypertensive drug, aliskiren confers some degree of renoprotection. On the other hand, RAAS inhibitors can affect renal hemodynamic mainly through dilation of the efferent arteriole. This can result in reduced intraglomerular pressure [35] and the decline in GFR, the reductions in GFR are presented by an increase in $\mathrm{sCr}$ levels. These changes can increase the risk of hyperkalemia and kidney injury occurring, especially in susceptible populations such as CKD (Chronic Kidney Disease), HF and diabetes mellitus [36].

However, our umbrella review identified limited evidence about aliskiren on cardiovascular and renal outcomes, being under-powered to reach certain conclusions. Most reviews available in the literature, comparing aliskiren with other drugs have focused on surrogate outcomes and did not provide robust estimates [37, 38]. Because most chronic CVD or CKD patients are already receiving standard treatment (ACE inhibitors or ARBs). Moreover, the addition of aliskiren would cause great harm to patients, many trails had to be stopped early. ALTITUDE trial [31] and ASTRONAUT trial [36] are two examples, in addition to standard theapy, aliskiren showed similar effects as placebo in cardiovascular and renal outcomes, rate of CVD death and HF hospitalization. In aliskiren group, there was a higher risk of adverse events including stroke, hyperkalemia, hypotension, and renal impairment/renal failure, allcause mortality was also found to be significantly increased in patients with diabetes but not in those without diabetes. As a consequence, the following ATMOSPHERE trial [39], which compared aliskiren to enalaprial in clinical outcomes in more than $7000 \mathrm{HF}$ patients, was also forced to remove those co-diabetes patients, to minimize possible harm.

Results of our umbrella review are in line with the current guide recommendations. In the latest ACC/AHA guideline [1], DRI aliskiren is classified as one of the effective oral antihypertensive drugs. It is recommended to use once a day, 150-300 mg each time. It is very long acting. After using aliskiren, there is an increased risk of hyperkalemia in CKD or in those on $\mathrm{K}^{+}$supplements or $\mathrm{K}^{+}$- sparing drugs. The guideline also pointed out that aliskiren may cause acute renal failure in patients with severe bilateral renal artery stenosis. In the latest ESC/ ESH guideline [40], aliskiren was emphasized again that it should be abandoned in pregnancy, it should not be combined with ACEIs or ARBs. In particular, we should be specially noted about contraindications and warnings for aliskiren-containing medicines in patients with diabetes, heart diseases and kidney problems.

\section{Strengths and limitations}

This umbrella review manifests the most comprehensive neutral evaluation of the literature of published associations between AM and related clinical outcomes. When possible, we reanalysed meta-analysis with a comprehensive approach that included the use of random effects analysis, the calculation of predicative intervals and publication bias, to get a better comparison across studies. Another advantage of our study is the stratification of some comparisons by dosage or category of the medication, to make the results more detailed and comprehensive. We also used the latest standard approach (AMSTAR 2) to evaluate quality of included reviews.

However, some unavoidable limitations and caveats are existed in our study. Firstly, in terms of addressing 
cardiovascular and renal effects, systematic reviews that studied these ranges are number limited or included small participants. Secondly, most of them examined the changes of surrogate markers. No hard outcomes like mortality, major cardiovascular or renal outcomes were examined. Secondly, though we had looked into the original studies of each systematic review, some unpublished data may still be missing, might lead to bias in our overview. Finally, due to the limited number of meta-analyses that studied the association between AM and clinical outcomes, some of included studies were not of high quality, especially the three pooled analyses.

\section{Conclusion}

AM has been investigated for associations with clinical efficacy and safety for the past recent years. We found solid evidence to support the benefits of aliskiren in the treatment of essential hypertension, it can produce significant effects of lowering BP and reliable safety. However, aliskiren presented comparable effects as placebo, other active comparators (ARBs, ACEIs and HCT) in cardiovascular and renal outcomes.

On the one hand, to draw firmer conclusions, we need more RCTs that studied deeply in aliskiren's efficacy on cardiovascular and renal outcomes, we need studies that can provide real hard outcomes and standardized reporting of analyse. On the other hand, our study is of great clinical value, it will help clinicians and patients weigh up the pros and cons of aliskiren for different diseases, for different types of patients.

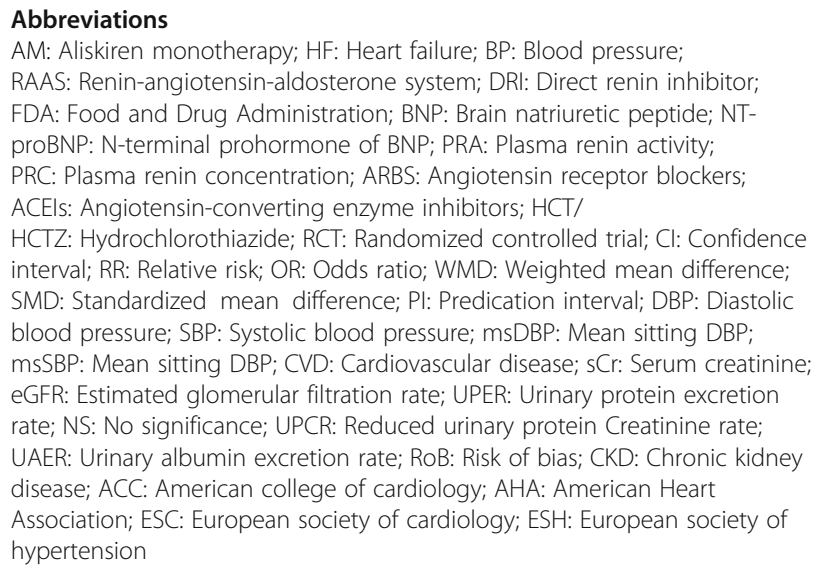

\section{Acknowledgments}

In particular, we wanted to thank Anne Folta Fish, an associate professor of Nursing College of Nursing at UM-St.Louis, who helped us to review and amend the manuscript voluntarily. Her suggestions are of great value and importance to us.

\section{Authors' contributions}

JS carried out the conception and design of the article. The implementation and feasibility analysis of research was conducted by JS; QZ and JL collected and sorted out the data. QZ, QJ and YW analyzed and explained the results, QZ wrote the thesis; JS reviewed and amended the draft of the article. QZ and JS supervised, mangaged and be responsible for the whole article. All authors read and approved of the final manuscript.

\section{Funding}

This work was supported by the Department of Education of Zhejiang Province, China [Grant numbers Y201635273]. The funding body has no role in the design of the study, data collection, analysis, and interpretation of data or in writing the manuscript.

\section{Availability of data and materials}

All data used for the umbrella review is contained within the manuscript.

Ethics approval and consent to participate

Not applicable.

\section{Consent for publication}

Not applicable.

\section{Competing interests}

All our authors report no conflicts of interest in this work.

Received: 6 December 2019 Accepted: 19 March 2020

Published online: 17 April 2020

\section{References}

1. Whelton PK, Carey RM, Aronow WS, Casey DE Jr, Collins KJ, Dennison Himmelfarb C, et al. 2017 ACC/AHA/AAPA/ABC/ACPM/AGS/APhA/ASH/ ASPC/NMA/PCNA guideline for the prevention, detection, evaluation, and Management of High Blood Pressure in adults: a report of the American College of Cardiology/American Heart Association task force on clinical practice guidelines. Hypertension. 2018;71(6):e13-e115. https://doi.org/10. 1161/HYP.0000000000000065.

2. Muntner P, Carey RM, Gidding S, Jones DW, Taler SJ, Wright JT Jr, et al. Potential U.S. population impact of the 2017 ACC/AHA high blood pressure guideline. J Am Coll Cardiol. 2018;71(2):109-18. https://doi.org/10.1016/j.jacc. 2017.10.073.

3. Egan BM, Li J, Hutchison FN, Ferdinand KC. Hypertension in the United States, 1999 to 2012: progress toward healthy people 2020 goals. Circulation. 2014;130(19):1692-9. https://doi.org/10.1161/CIRCULATIONAHA. 114.010676

4. Vos T, Flaxman AD, Naghavi M, Lozano R, Michaud C, Ezzati M, et al. Years lived with disability (YLDs) for 1160 sequelae of 289 diseases and injuries 1990-2010: a systematic analysis for the global burden of disease study 2010. Lancet. 2012;380(9859):2163-96. https://doi.org/10.1016/s01406736(12)61729-2.

5. Zaman MA, Oparil S, Calhoun DA. Drugs targeting the renin-angiotensinaldosterone system. Nat Rev Drug Discov. 2002;1(8):621-36. https://doi.org/ 10.1038/nrd873.

6. Skeggs LT Jr, Kahn JR, Lentz K, Shumway NP. The preparation, purification, and amino acid sequence of a polypeptide renin substrate. J Exp Med. 1957;106(3):439-53.

7. rao MS. Inhibition of the renin Angiotensin Aldosterone System: focus on Aliskiren. JAPI. 2010;58:102-8.

8. Musini VM, Lawrence KA, Fortin PM, Bassett K, Wright JM. Blood pressure lowering efficacy of renin inhibitors for primary hypertension. Cochrane Database Syst Rev. 2017;4:Cd007066. https://doi.org/10.1002/14651858. CD007066.pub3

9. Makani H, Bangalore S, Desouza KA, Shah A, Messerli FH. Efficacy and safety of dual blockade of the renin-angiotensin system: meta-analysis of randomised trials. Bmj. 2013;346(jan28 1):f360. https://doi.org/10.1136/ bmj.f360.

10. Nijst $P$, Verbrugge FH, Martens $P$, Bertrand PB, Dupont M, Francis GS, et al. Plasma renin activity in patients with heart failure and reduced ejection fraction on optimal medical therapy. J Renin-Angiotensin-Aldosterone Syst. 2017;18(3):1470320317729919. https://doi.org/10.1177/1470320317729919.

11. Braam B, Koomans $H$. Renal responses to antagonism of the reninangiotensin system. Curr Opin Nephrol Hypertens. 1996:5:89-96. https://doi. org/10.1097/00041552-199601000-00015.

12. Riley RD, Higgins JPT, Deeks JJ. Interpretation of random effects metaanalyses. BMJ. 2011;342:d549. https://doi.org/10.1136/bmj.d549. 
13. JPT H, Thompson SG, JPT H. Thompson SGQuantifying heterogeneity in a meta-analysis. Stat Med. 2002;21:1539-58.

14. Shea BJ, Reeves BC, Wells G, Thuku M, Hamel C, Moran J, et al. AMSTAR 2: a critical appraisal tool for systematic reviews that include randomised or non-randomised studies of healthcare interventions, or both. BMJ. 2017;358: j4008. https://doi.org/10.1136/bmj.j4008.

15. Chen Y, Meng L, Shao H, Yu F. Aliskiren vs. other antihypertensive drugs in the treatment of hypertension: a meta-analysis. Hypertens Res. 2013;36(3): 252-61. https://doi.org/10.1038/hr.2012.185.

16. Gao D, Ning N, Niu X, Wei J, Sun P, Hao G. Aliskiren vs. angiotensin receptor blockers in hypertension: meta-analysis of randomized controlled trials. Am J Hypertens. 2011;24(5):613-21. https://doi.org/10.1038/ajh.2011.3.

17. Zhenfeng Z, Huilan S, Junya J, Dong L, Shan L. A systematic review and meta-analysis of aliskiren and angiotension receptor blockers in the management of essential hypertension. J Renin-Angiotensin-Aldosterone Syst. 2011;12(2):102-12. https://doi.org/10.1177/1470320310381912.

18. Verdecchia P, Angeli F, Mazzotta G, Ambrosio G, Reboldi G. Angiotensin converting enzyme inhibitors and angiotensin receptor blockers in the treatment of hypertension: should they be used together? Curr Vasc Pharmacol. 2010;8(6):742-6

19. Powers B, Greene L, Balfe LM. Updates on the treatment of essential hypertension: a summary of AHRQ's comparative effectiveness review of angiotensin-converting enzyme inhibitors, angiotensin II receptor blockers, and direct renin inhibitors. J Manag Care Pharm. 2011;17(8 Suppl):S1-14.

20. Stanton AV, Gradman AH, Schmieder RE, Nussberger J, Sarangapani R, Prescott MF. Aliskiren monotherapy does not cause paradoxical blood pressure rises: meta-analysis of data from 8 clinical trials. Hypertension. 2010; 55(1):54-60. https://doi.org/10.1161/hypertensionaha.109.135772.

21. Luo Y, Chen Q. Efficacy of aliskiren supplementation for heart failure : a meta-analysis of randomized controlled trials. Herz. 2018. https://doi.org/10. 1007/s00059-018-4679-1.

22. Zhang JT, Chen KP, Guan T, Zhang S. Effect of aliskiren on cardiovascular outcomes in patients with prehypertension: a meta-analysis of randomized controlled trials. Drug Des Devel Ther. 2015;9:1963-71. https://doi.org/10. 2147/dddt. S75111.

23. Zheng SL, Roddick AJ, Ayis S. Effects of aliskiren on mortality, cardiovascular outcomes and adverse events in patients with diabetes and cardiovascular disease or risk: a systematic review and meta-analysis of 13,395 patients. Diab Vasc Dis Res. 2017;14(5):400-6. https://doi.org/10.1177/ 1479164117715854.

24. White WB, Bresalier R, Kaplan AP, Palmer BF, Riddell RH, Lesogor A, et al. Safety and tolerability of the direct renin inhibitor aliskiren in combination with angiotensin receptor blockers and thiazide diuretics: a pooled analysis of clinical experience of 12,942 patients. J Clin Hypertens (Greenwich). 2011; 13(7):506-16. https://doi.org/10.1111/j.1751-7176.2011.00438.x.

25. Louvis N, Coulson J. Renoprotection by direct renin inhibition: a systematic review and Meta- analysis. Curr Vasc Pharmacol. 2018;16(2):157-67. https:// doi.org/10.2174/1570161115666170502104809

26. Rizos EC, Agouridis AP, Elisaf MS. Aliskiren in patients with diabetes: a systematic review. Curr Vasc Pharmacol. 2012;10(2):140-6.

27. Lambers Heerspink HJ, Perkovic V, de Zeeuw D. Renal and cardio-protective effects of direct renin inhibition: a systematic literature review. J Hypertens. 2009:27(12):2321-31. https://doi.org/10.1097/HJH.0b013e3283310f92.

28. Latini R, Masson S, Wong M, Val-HeFT investigators. Incremental prognostic value of changes in B-type natriuretic peptide in heart failure. Am J Med. 2006;119(1):70

29. Pantzaris ND, Karanikolas E, Tsiotsios K, Velissaris D. Renin Inhibition with Aliskiren: A Decade of Clinical Experience. J Clin Med. 2017;6:6. https://doi. org/10.3390/jcm6060061.

30. McMurray JJ, Krum H, Abraham WT, Dickstein K, Kober LV, Desai AS, et al. Aliskiren, Enalapril, or Aliskiren and Enalapril in heart failure. N Engl J Med. 2016;374(16):1521-32. https://doi.org/10.1056/NEJMoa1514859.

31. Parving HH, Brenner BM, McMurray JJ, de Zeeuw D, Haffner SM, Solomon $\mathrm{SD}$, et al. Cardiorenal end points in a trial of aliskiren for type 2 diabetes. $N$ Engl J Med. 2012;367(23):2204-13. https://doi.org/10.1056/NEJMoa1208799.

32. Okada Y, Shibata S, Fujimoto N, Best S, Levine B, Fu Q. Long-term effects of a renin inhibitor versus a thiazide diuretic on arterial stiffness and left ventricular diastolic function in elderly hypertensive patients. Am J Phys Regul Integr Comp Phys. 2017;313:ajpregu.00125.2017. https://doi.org/10. 1152/ajpregu.00125.2017.
33. Okada Y, Shibata S, Fujimoto N, Best S, Levine BD, Fu Q. Long-term effects of a renin inhibitor versus a thiazide diuretic on arterial stiffness and left ventricular diastolic function in elderly hypertensive patients; 2017.

34. Rashid HU. Renoprotection, renin inhibition, and blood pressure control: the impact of aliskiren on integrated blood pressure control. Integr Blood Press Control. 2010;3:133-44. https://doi.org/10.2147/IBPC.S12407.

35. Coffman TM, Crowley SD. Kidney in hypertension: Guyton redux. Hypertension. 2008;51(4):811-6. https://doi.org/10.1161/HYPERTENSIONAHA. 105.063636.

36. Gheorghiade M, Böhm M, Greene S, Fonarow G, Lewis E, Zannad F, et al. Effect of Aliskiren on Postdischarge mortality and heart failure readmissions among patients hospitalized for heart failure the ASTRONAUT randomized trial. JAMA. 2013;309:1-11. https://doi.org/10.1001/jama.2013.1954.

37. Makani H, Bangalore S, Desouza K, Shah A, Fh M. Efficacy and safety of dual blockade of the renin-angiotensin system: Meta-analysis of randomised trials. BMJ Br Med J. 2013;346:f360. https://doi.org/10.1136/bmj.f360.

38. Sanders GD, Coeytaux R, Dolor RJ, Hasselblad V, Patel UD, Powers B, et al. AHRQ Comparative Effectiveness Reviews. In: Angiotensin-Converting Enzyme Inhibitors (ACEls), Angiotensin II Receptor Antagonists (ARBs), and Direct Renin Inhibitors for Treating Essential Hypertension: An Update. Rockville: Agency for Healthcare Research and Quality (US); 2011.

39. Krum H, Massie B, Abraham W, Dickstein K, Køber L, McMurray J, et al. Direct renin inhibition in addition to or as an alternative to angiotensin converting enzyme inhibition in patients with chronic systolic heart failure: rationale and design of the Aliskiren trial to minimize Outcomes in patients with HEart failuRE (ATMOSPHERE) study. Eur J Heart Fail. 2011;13:107-14. https:// doi.org/10.1093/eurjhf/hfq212.

40. Williams B, Mancia G, Spiering W, Agabiti Rosei E, Azizi M, Burnier M, et al. 2018 ESC/ESH guidelines for the management of arterial hypertension. Eur Heart J. 2018;39(33):3021-104. https://doi.org/10.1093/eurheartj/ehy339.

\section{Publisher's Note}

Springer Nature remains neutral with regard to jurisdictional claims in published maps and institutional affiliations.

Ready to submit your research? Choose BMC and benefit from:

- fast, convenient online submission

- thorough peer review by experienced researchers in your field

- rapid publication on acceptance

- support for research data, including large and complex data types

- gold Open Access which fosters wider collaboration and increased citations

- maximum visibility for your research: over $100 \mathrm{M}$ website views per year

At $\mathrm{BMC}$, research is always in progress.

Learn more biomedcentral.com/submissions 